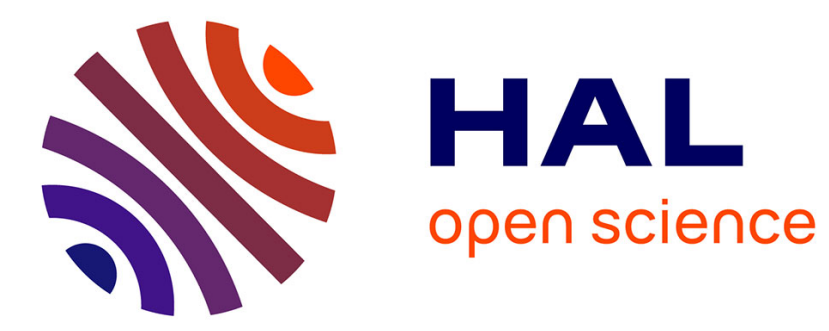

\title{
Subflorescence and plaster drying dynamics
}

Mamadou Diaga Seck, Emmanuel Keita, Pamela Françoise Faure, Pierrick

Cavalié, Maxime van Landeghem, Stéphane Rodts, Philippe Coussot

\section{To cite this version:}

Mamadou Diaga Seck, Emmanuel Keita, Pamela Françoise Faure, Pierrick Cavalié, Maxime van Landeghem, et al.. Subflorescence and plaster drying dynamics. Chemical Engineering Science, 2016, 148, pp.203 - 211. 10.1016/j.ces.2016.04.012 . hal-01784858

\section{HAL Id: hal-01784858 \\ https://hal-enpc.archives-ouvertes.fr/hal-01784858}

Submitted on 3 May 2018

HAL is a multi-disciplinary open access archive for the deposit and dissemination of scientific research documents, whether they are published or not. The documents may come from teaching and research institutions in France or abroad, or from public or private research centers.
L'archive ouverte pluridisciplinaire HAL, est destinée au dépôt et à la diffusion de documents scientifiques de niveau recherche, publiés ou non, émanant des établissements d'enseignement et de recherche français ou étrangers, des laboratoires publics ou privés. 


\title{
Subflorescence and plaster drying dynamics
}

\author{
Mamadou Diaga Seck ${ }^{a, b}$, Emmanuel Keita $^{c}$, Paméla Faure ${ }^{a}$, Pierrick Cavalie ${ }^{b}$, Maxime Van \\ Landeghem ${ }^{b}$, Stéphane Rodts ${ }^{a}$, Philippe Coussot ${ }^{a^{*}}$ \\ a Université Paris-Est, Laboratoire Navier, ENPC-IFSTTAR-CNRS, 2 allée Kepler, 77420 Champs-sur- \\ Marne, France \\ Saint-Gobain Recherche, 39 Quai Lucien Lefranc, BP135, 93303 Aubervilliers Cedex, France \\ ' Université Paris-Est, MAST, FM2D, IFSTTAR, 14-20 Bd Newton, 77420 Champs-sur-Marne, France \\ * Corresponding author
}

\begin{abstract}
The dynamics of plaster drying and the impact of subflorescence on the process are described through Magnetic Resonance Imaging and X-Ray Microtomography measurements. It is shown that crystals deposit around the air-liquid interface the closest to the sample free surface, which induces a recession of this interface within the sample at a rate only depending on the current saturation (water to pore volume ratio). Thus the distribution of crystals deposited during evaporation essentially depends on the history of saturation. The drying dynamics then results from vapor diffusion through the less porous layers of crystal accumulation below the sample free surface. This in particular makes it possible to predict the dramatic decrease of the drying rate after successive imbibition-drying cycles.
\end{abstract}

Key words: Plaster; drying; subflorescence; precipitation, dewetting

\section{Introduction}

To obtain plasterboards so useful for modern constructions, there are two important phenomena that must take place during the manufacturing process: hydration, through which plaster paste passes from a pasty state to a solid state, and drying, through which the excess of the mixing water added for workability purposes is removed. The hydration reaction can be described as the successive dissolution of hemihydrates $\left(\mathrm{CaSO}_{4}, 1 / 2 \mathrm{H}_{2} \mathrm{O}\right.$, initial water-to-powder ratio of 0.8$)$ and precipitation of dihydrates $\left(\mathrm{CaSO}_{4}, 2 \mathrm{H}_{2} \mathrm{O}\right)$ in water [1-4]. Detailed information on these processes may be found in [5-6]. After this chemical reaction which lasts about 40 min without accelerators [7], a significant excess of water must be removed from the material, an operation which requires a lot of energy. Understanding the drying processes in plaster pastes should contribute to develop more efficient drying techniques and procedures, and to consume less energy. Here we focus on one of the technics used in manufacture to remove the residual water: by a convective air flow [8].

Existing knowledge on drying characteristics of plasters essentially concerns materials already in solid state which are imbibed then dried and tends to suggest [9] that as long as pure water is used such materials exhibit drying properties close to usual simple homogeneous porous media. A specific aspect has been studied in more details in recent years, namely the transport of salts during drying of solid plaster samples. It has been shown that salts are transported towards the free surface and then can either crystallize inside the plaster or move outside, towards the substrate [9]. It was also 
suggested that this salt crystallization leads to the formation of a structure inside the plaster, whose characteristics depend on the drying rate and in turn impact the drying rate [10].

The drying characteristics of plaster pastes just after their preparation has not been studied in depth so far whereas it is a critical step of the process of plasterboard production. Previous studies concerning plaster properties during its preparation focused on the structure evolution in time, in particular with the help of NMR, showing that the pore distribution evolves as a result of hydration [11-12]. A recent work finally showed that the drying rate of plaster pastes is significantly lower than that expected for a pure liquid evaporating from a simple homogeneous porous medium [13]. This effect was shown to be enhanced by the air flow velocity and the initial solid/water ratio. From further tests under various conditions it was suggested that this effect is due to the crystallization of gypsum ions below the sample free surface, which creates a dry region and decreases the drying rate by increasing the length of the path the vapor has to follow before reaching the free surface. The specificity of plaster drying is indeed that at the end of the initial setting process (due to precipitation of dihydrates) the interstitial solution is saturated with ions $\left(\mathrm{Ca}^{2+}, \mathrm{SO}_{4}{ }^{2-}\right)$, which can then precipitate as gypsum crystals and accumulate in some regions as soon as some water is extracted by evaporation. Further work is needed to understand the physical mechanisms and then to be able to predict the drying rate as a function of material characteristics and boundary conditions.

The understanding of this phenomenon is interesting for other reasons. Salt transport and crystallisation in building materials are responsible for major damages of modern structures [14] and cultural heritage [15]. Similar effects in rocks can alter $\mathrm{CO} 2$ injectivity [16] and are at the origin of various geomorpholocial processes [17]. For example ions dissolved during imbibition can then, during drying, reach the solubility limit and precipitate as crystals below (subflorescence) or above (efflorescence) the sample free surface. These crystals may clog the porous structure [18], lead to flaking and crumbling [19] or reduce the compression strength of the material [20]. The mechanisms at the origin of damages have been discussed: crystallization pressure, supersaturation, solid volume change from thenardite to mirabilite [21-25]; but it was also acknowledged that the remaining liquid films can play a significant role on crystallization processes [26]. Extensive studies $[17,27]$ showed the variety of trends observed depending on solution properties (salt type and additives), but it was finally pointed out [17, 28-29] from detailed information concerning salt and damage localization, that possible damages critically depend on the localization of salt crystals which requires the description of the dynamics of salt transport, spatial distribution and crystallization and its coupling with drying mechanisms.

For efflorescence the basic theoretical concepts of salt transport resulting from water flow during drying were confirmed by following the salt distribution by MRI [30-31]. It was then shown that crystals deposited at the sample free surface behave as a porous medium which pumps the liquid and brings ions around the most external liquid-air interface to form new crystals [32-33]. A recent study showed in addition that the structure of the efflorescence layer can evolve with successive imbibition-drying cycles, which successively modifies the drying rate, increasing or decreasing it depending on the crystallization pathways or on the humidity of the crust [34]. Microtomography observations of the three phases around the sample top provided a detailed description of the process, which makes it possible to establish a model of crust formation dynamics as a function of drying rate [35]. Besides it was shown that some unexpected effects (in drying rate variations) can result from the interplay between the crust and the evaporation conditions [36-37].

Although it is a more damaging effect than efflorescence much less is known concerning the local mechanisms of subflorescence. It was shown from X-Ray microtomography that subtle local effects occur essentially at the scale of the pore [38], and tend to induce pore clogging which reduces the drying rate, but these effects depend of the type of salt at work [18]. From original experiments in 2D 
system it was also shown that a specific subflorescence pattern can grow because the crystal structure form a porous media which can drain a saturated salty solution up to the evaporating front, which eventually causes the drying rate to increase [39]. Recently the work of Derluyn with the help of neutron radiography and X-ray microtomography provided new insights on salt transport, accumulation, and induced damage in limestone samples [40-41]. However our knowledge of the relationship between subflorescence and drying dynamics in porous media is still limited, essentially because it is more difficult to get a direct view of the processes at a local scale. Yet it governs the spatial distribution of crystals which in turn determines the position of possible damages, and this knowledge would open the way to the development of relevant tools for controlling or avoiding such effects. Taking advantage of MRI (Magnetic Resonance Imaging) data such an approach was recently developed for predicting the accumulation of initially suspended colloidal particles and its impact on drying dynamics [42].

Here we aim at studying in more detail the process of subflorescence in plaster. From repeated imbibition-drying cycles on the same sample we observe that the drying rate is dramatically decreased as a result of crystal accumulation inside the sample. Through MRI and X-Ray microtomography we show that crystals are deposited around the first (i.e. most external) air-liquid interface, which induces its recession in the sample at a rate only depending on the current saturation (water to pore volume ratio) and the current total liquid volume in the sample. From this information we deduce a simple phenomenological model which predicts the drying dynamics of plaster under different conditions.

\section{Materials and methods}

We used a commercial $\alpha$-plaster (Saint-Gobain Recherche, France) with a purity higher than $95 \%$ wt, a specific surface of $7500 \mathrm{~g} / \mathrm{m}^{2}$ (measured with the Blaine air permeability test), and a grain size between 10 and $20 \mu \mathrm{m}$. The impurities are mainly clay (colloidal) particles. Our plaster paste samples are prepared according to a well-defined mixing protocol $[13,43]$. The initial water to powder ratio $(w / p)$, i.e. the ratio of the mass of water to the mass of powder, was between 0.5 and 0.8 . At the end of the hydration reaction (after about $25 \mathrm{~min}$, as found from NMR measurements [13]) which consumed about $20 \%$ of the initial water mass, the solid structure is filled with a calcium - sulphate solution at the gypsum solubility limit ( $\psi=2.65 \mathrm{~g} / \mathrm{l}$ ). This porous medium has initially a homogeneous porosity of $58 \%$ and a bimodal pore size distribution (from Mercury Intrusion Porosimetry) with peaks at 0.6 and 5 microns or so.

The plaster pastes are finally poured into a cylindrical container ( $35 \mathrm{~mm}$ or $54 \mathrm{~mm}$ diameter, and different heights from 12 to $40 \mathrm{~mm}$ ) just at the end of the mixing protocol (i.e. initial time). The sample set up is then immediately dried by exposing its (open) top free surface to a tangential stream of dehydrated air (relative humidity $<1 \%)$ at ambient temperature $\left(23^{\circ} \mathrm{C} \pm 1\right)$ through a long rectangular conduit of $60 \times 3 \mathrm{~mm}^{2}$ section. The sample has its free surface appearing from a hole in the continuity of the channel wall, and lies on a scale so that its mass is followed in time. The saturation $\phi$ is computed from the ratio of the current water content to the initial water mass. This initial water mass is deduced from complete drying at $45^{\circ} \mathrm{C}$ after the test. Note that we choose this temperature of drying to avoid any possibility of decomposition of gypsum as it was indeed shown [43] that at such temperature the chemical structure of the hydrates is unchanged. The drying dynamics is well described through the drying rate, i.e. $\mathrm{d} \phi / \mathrm{d} t$, as a function of $\phi$. Such data are reproducible with an uncertainty of about $20 \%$ on the drying rate [13] in particular due to the slight 
variations of the surface shape of the samples and the data processing to compute $\mathrm{d} \phi / \mathrm{d} t$ from experimental data.

In order to look at the water distribution inside the sample in time during the test we inserted the whole drying set-up (without the scale) at the center of the magnet of a vertical proton MRI (Magnetic Resonance Imaging) system (Avance 24/80 DBX by Bruker, 0.5T superconducting magnet by Oxford, $20 \mathrm{~cm}$ inner diameter). The distribution of apparent water along the sample axis was measured using a one-dimensional double spin-echo sequence, i.e. two first echoes of the so-called Carr-Purcell-Meibom-Gill sequence [44-46] with extrapolation to compensate spin-spin relaxation. Each measured value of this distribution corresponds to the total amount of water in a thin crosssectional layer 98 microns thick situated at a given position along the sample vertical axis. To get a satisfactory signal to noise ratio, the measurement time for each profile was two hours, so that a profile represents the average water distribution over that duration. Profile measurements were repeated every two hours.

In order to know how the internal structure evolves as a result of drying we used a $R X$-Solutions EasyTom tomograph equipped with an X-ray polychromatic microfocus source with a spot size of 5 $\mu \mathrm{m}(6,4 \mathrm{~W}$ and $80 \mathrm{Kev})$ allowing a 3D imaging of the structure with cubic volume element of $5.8 \mu \mathrm{m}$ edge.

\section{Results}

During the first drying after preparation the drying rate progressively decreases at the beginning of the desaturation then more rapidly decreases below $\phi \approx 0.15$ (see Figure 1). As already explained in [13] this result contrasts with the well-known effect of constant drying rate down to a saturation of the order of $10 \%$ (at sufficiently low air flow velocity) for a simple porous medium filled with a pure hydrophilic liquid [47-48]. Indeed, during drying simple materials exhibit a first regime, called the CRP (Constant Rate Period), during which the drying rate is approximately constant. During this period the saturation remains uniform in the material and the evaporation occurs from the upper liquid films very close to the free surface of the sample, while the liquid is drained by capillary effects through the film network throughout the sample. Then the drying rate starts to decrease significantly, this is the Falling Rate Period (FRP). In that regime the liquid flow driven by basic capillary effects is unable to provide liquid at a sufficient rate at the sample free surface, so that a thin dry region forms in this region which lowers the drying rate (since now the vapor has to diffuse through this region before reaching the sample free surface). The size of this dry region slightly increases during this regime. Finally a last regime takes place when the dry region now increases widely because there is no longer any possibility for the liquid to be drained towards the sample free surface. 


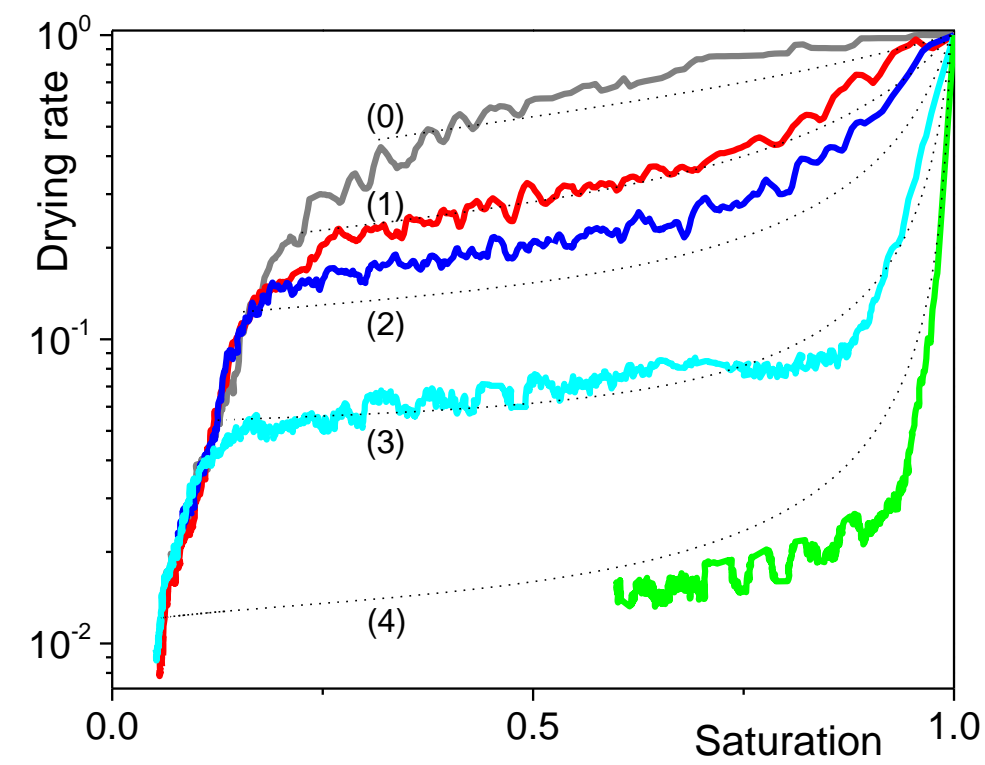

Figure 1: Drying rate (rescaled by the initial one, i.e. $2.8 \mathrm{~g} / \mathrm{hr}$ ) as a function of saturation for the plaster for an increasing number (from top to bottom, numbers in brackets) of imbibition-drying cycles after the paste preparation (sample height $40 \mathrm{~mm}, w / p=0.8$, air flux velocity: $7.3 \mathrm{~m} / \mathrm{s}$ ): (continuous lines) experiments, (dotted lines) model (equation 2) with $\alpha=48 \times 10^{4}$ and $\beta=4000 \mathrm{~g}^{-1}$. A drying process should thus be followed from right to left.

Note that this effect of decrease of the drying rate observed with a plaster paste is not due the hydration reaction since after a relatively short time with regards to the total duration of the drying test (typically 40 hours) the hydration reaction ends, and the solid structure is fixed [13]. Here the main difference between our sample and a simple porous medium as above described is the presence of ions $\left(\mathrm{Ca}^{2+}, \mathrm{SO}_{4}{ }^{2-}\right)$ in the interstitial solution, which can precipitate as gypsum crystals and accumulate in some regions, thus reducing the permeability and the drying rate. Since the sample thickness remains constant and we do not see significant accumulation of crystals above the free surface, this means that we are dealing here with subflorescence. During most of the initial period of drying the liquid flows towards the regions of evaporation, which are mainly around the air-liquid interfaces the closest to the sample free surface because the air in the regions below is almost saturated in vapour [48]. The ions contained in the liquid are thus globally transported towards the free surface where the slight concentration increase due to each evaporation increment will readily induce crystallisation since the solution is at its solubility limit. This implies that we have a reduction of the pore size or the porosity at least in the upper regions of the sample. In porous media however, such effects usually only slightly reduce the duration of the initial constant rate period [47-48]; indeed in this regime the rate of evaporation is imposed by the external air flow as long as the liquid can flow sufficiently rapidly through the medium towards the sample free surface as a result of capillary effects. This is so because the thickness of such a layer of small pores is so small that the pressure gradient imposed by capillary effects are easily extremely large and can easily move water through it. This means that in the present case something different, or more precisely some additional effect, happens. 
In order to better appreciate the effect we repeated the test several times with the same sample after the initial drying. Our procedure consisted to first immerse the sample in a calcium - sulfate saturated solution, then put this system under vacuum. Under such conditions it seems unlikely that the initially solid gypsum can decompose, as can be observed under other conditions [49], since it is not directly in contact with a gas at low pressure and instead is in contact with a saturated solution. Thus the initial system at the beginning of each new drying process was a sample filled with a calcium - sulphate saturated water solution. This situation differs from that of Desarnaud et al. [34] in which pure water is re-introduced in the porous sample (sandstone) and possible salt dissolution depended on the exact impregnation procedure.

All cycles share the same initial drying rate (see Figure 1) since each sample is saturated at the beginning of the test so that at the initial conditions of evaporation under the same flux are similar. On the contrary drying rates observed after a short while are significantly lower for an increasing number of cycles, so that after five imbibition-drying cycles, for $\phi$ in the range [0.15-0.8] the drying rate reaches values almost two orders of magnitude lower than the initial one (see Figure 1). It is remarkable that this dramatic decrease in drying rate is obtained with a total (after the five cycles) precipitated gypsum to sample mass ratio of $0.24 \%$. We also remark that after the first imbibitiondrying cycle the shape of the drying curve tends to a strong initial decrease followed by a plateau and finally a new strong decrease (see Figure 1). This suggests that the conditions of evaporation widely vary, which should be visible in the liquid distribution.

We can get further information from MRI experiments providing the evolution of the water distribution along the sample axis. At first sight (see Figure 2a) the results for the first cycle do not differ from those typically observed in simple porous media [13]. The water distribution apparently remains homogeneous in the sample during a long first period (down to saturation of the order of $30 \%$ ) (see Figure $2 a$ ), then we can see a dry front receding inside the sample while the plateau level behind goes on decreasing. Similar results are obtained for the subsequent cycles (see Figure $2 b$ ), although the time to reach each curve increases with the cycle number and some region of lower saturation forms at the beginning of drying below the sample free surface. This region of thickness much larger than the zone of accumulation of crystals is likely due to some heterogeneity of the sample. It does not evolve further during the subsequent cycles, and does not play any role in the evolution of the drying rate discussed in this paper.

The second stage of evolution of these profiles, i.e. large receding front, corresponds to the saturation range for which we have the second fast decrease of the drying. In this regime the liquid flow under capillary effects is not sufficiently rapid compared to the evaporation imposed by air flow along the sample free surface. The point is that in contrast, in the first stage the homogeneous saturation is usually associated with the constant rate period [50], which is not the case here, in particular with the dramatic decrease in drying rate for the last cycles. There is thus an effect, which is not just a dry front of significant thickness, which remains to be found to explain this drying rate decrease. 

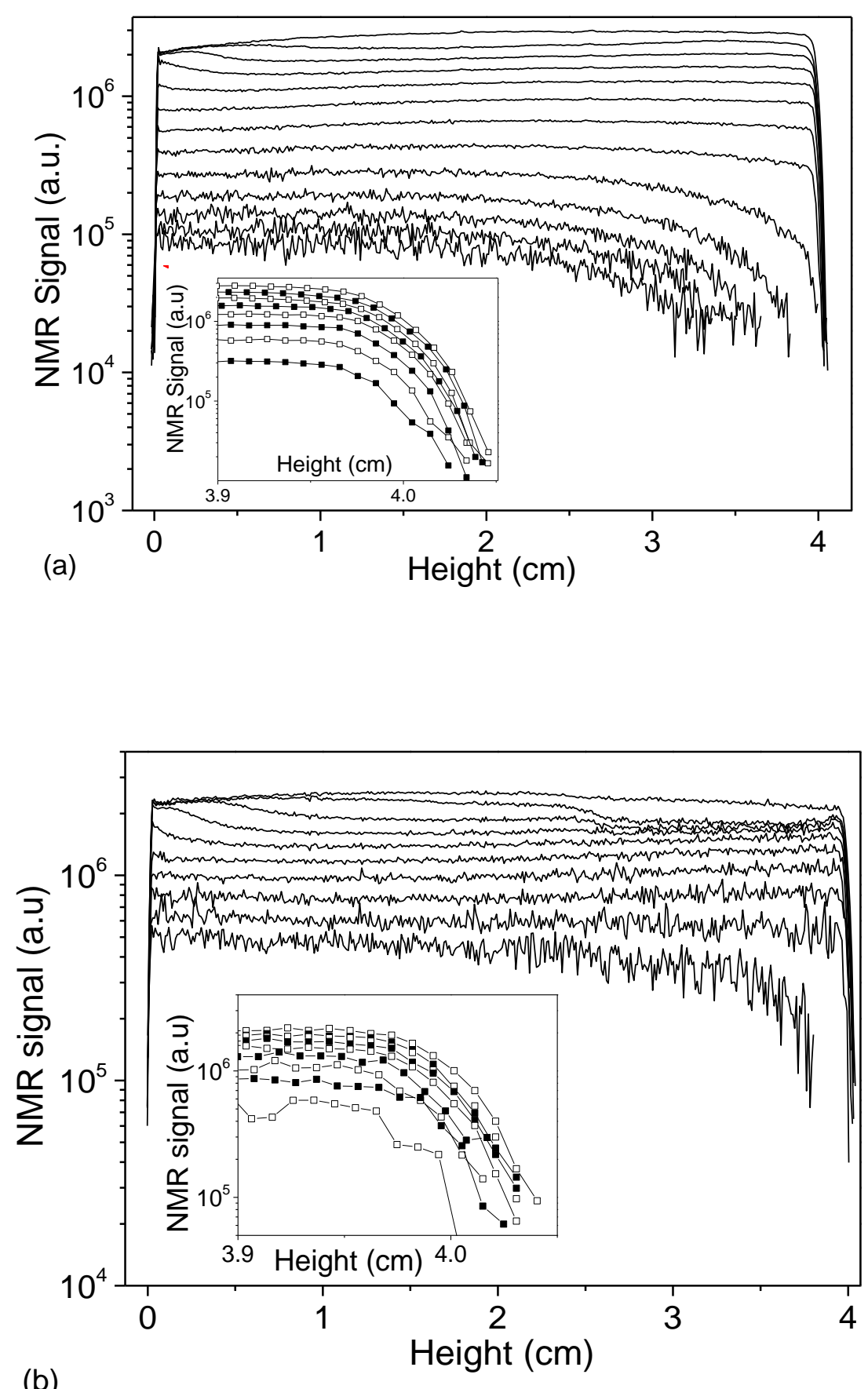

Figure 2: Water distribution profiles in a plaster at successive times (every two hours from top to bottom) during the first drying after preparation (a) and after the first cycle (b). The experimental conditions are those described in Figure 1 . The insets show a zoom on these profiles around the sample free surface.

This suggests that some evolution of the porous structure plays a role. The aspect of the structure at different depths from the free surface as observed by X-ray microtomography is shown in Figure 3. These images are maps of the attenuation coefficient for X-Rays in a thin layer of thickness $5.8 \mu \mathrm{m}$ situated at a given position along the sample axis. In such pictures, since initial solid matrix and subsequently deposited crystals are of the same chemical composition and density they exhibit the 
same X-ray attenuation coefficient, so that the grey scale is linearly related to the porosity, i.e. black for a void volume of thickness larger than $5.8 \mu \mathrm{m}$, white for a pure solid volume. At depths beyond a few hundreds of microns the structure keeps the aspect shown in Figure $3 c$, with a large number of relatively dark regions of various size, which indicates a large number of voids. At the approach of the free surface the number of dark regions decreases, while the amount of bright regions is higher; this indicates that the becomes globally less porous (see Figure 3a,b), the pores apparently become smaller and less numerous, an effect which obviously results from the deposition of gypsum crystals.

In order to quantify this effect we computed the average porosity for each image along the vertical direction. The examples of Figure 4 show that after a rapid decrease, which corresponds to the crossover of the sample roughness, the porosity reaches a minimum value associated with a high concentration of crystals, then it increases rapidly and reaches a plateau. The deposition of crystals occurs preferentially where the evaporation occurs, since, there, by water removal, the critical dissolution concentration tends to be overcome first, and from MRI we know that most of the evaporation occurs close to the free surface so that most crystals are formed around the sample top. Some crystallization may occur while the receding front develops over large depths inside the sample but in that case the remaining ion content is small and the crystals will be dispersed over a large distance. We deduce that the plateau essentially corresponds to the unchanged porous structure.

The porosity curves vary from one cycle to another essentially by a variation of the peak (minimum) size, which increases for increasing cycle number. This result is qualitatively close to that obtained by Derluyn et al. [40] with sodium sulfate crystalizing in drying limestone. This means that the pores are filled more and more at each step, essentially below the sample free surface. Note that the curve for the third cycle does not exhibit a shape quite consistent with the general evolution of the other curves. This is likely due to the uncertainty on data (which is hard to quantify). More generally, considering the (complex) way these data are obtained we cannot consider that they provide precise values of the effective porosity, the most relevant results concern the variations of this apparent porosity with the depth in the sample. As a consequence, in the following we will essentially focus of these variations (through a probability density function on crystal location, see below).

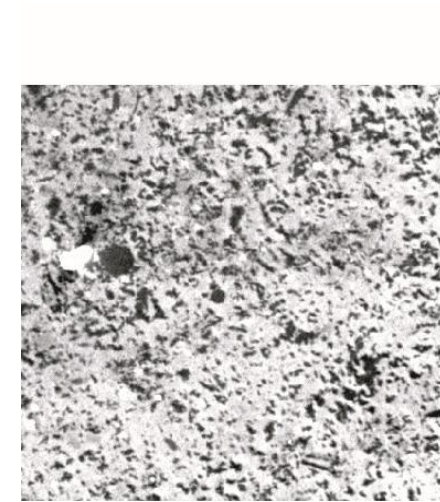

(a)

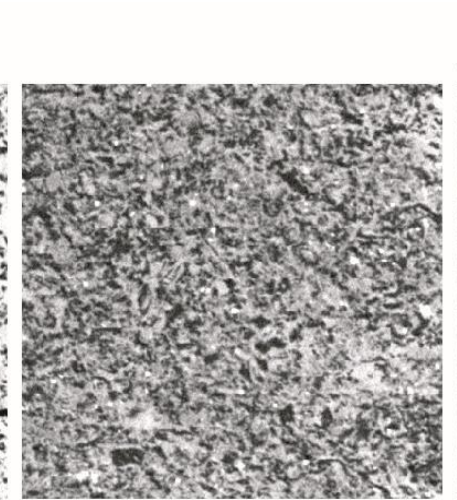

(b)

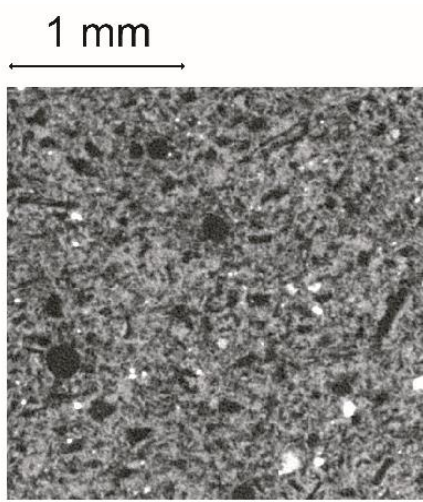

(c)

Figure 3: Views from tomography (with the same contrast) of the structure of the plaster sample after the $4^{\text {th }}$ cycle at different depths $(x)$ from the free surface: (a) $40 \mu \mathrm{m}$, (b) $190 \mu \mathrm{m}$, (c) 1.7 $\mathrm{mm}$. The experimental conditions correspond to those of Figure 1. 


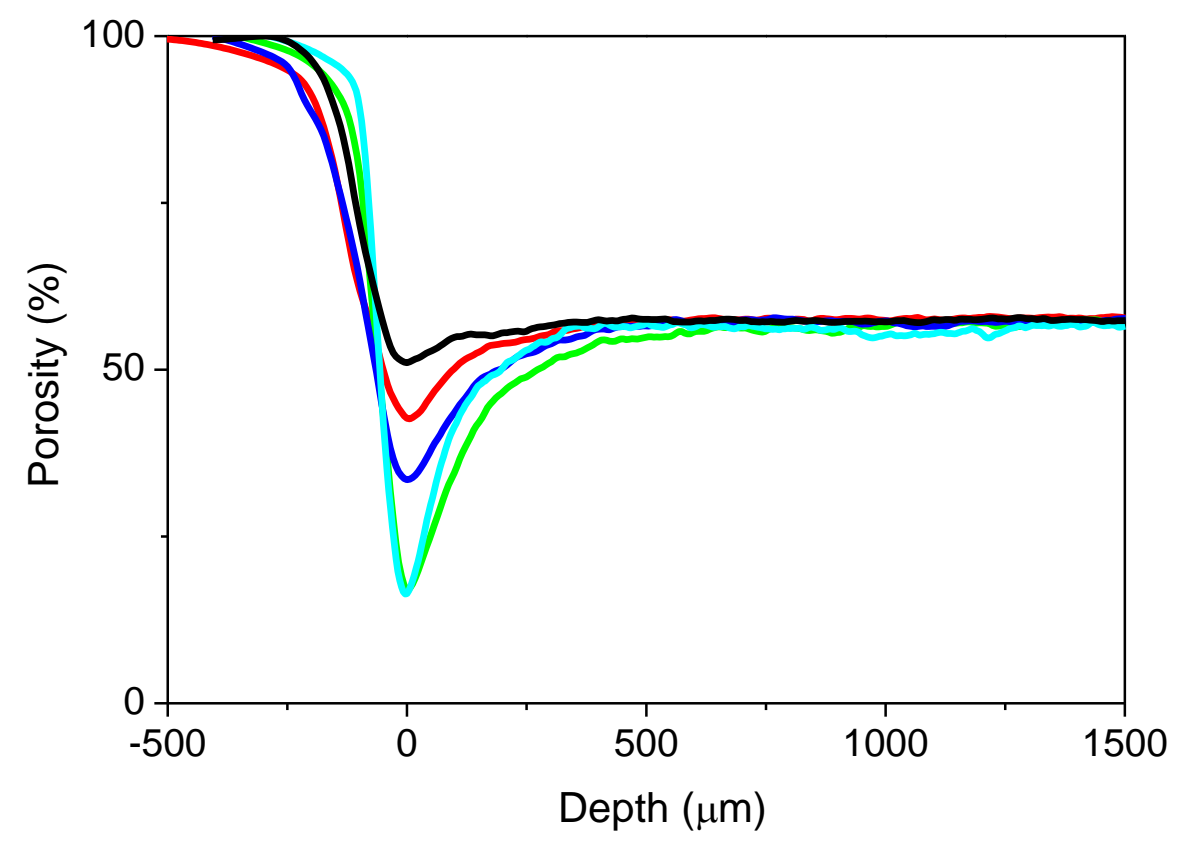

Figure 4: Average porosity (as deduced from X-Ray tomography) in elementary cross-sectional layers along the sample axis as a function of the depth in the sample for the successive dryingimbibition cycles (same conditions and colors as in Figure 1).

\section{Discussion}

3.1 General data analysis

In order to look consistently at the evolution of the profiles of porosity as obtained in Figure 4 we can withdraw the plateau level from each of these curves, and rescale them by their integral. Note that in this representation we choose the initial abscissa as the mid position in the sample roughness. The resulting curves (see Figure 5) are proportional to the probability density function ( $p$ ) on crystal location, formed since the beginning of the series of cycles, along the downward vertical direction ( $x$ ). Thus the probability to have a crystal between $x$ and $x+d x$ is $p(x) d x$. Finally, it appears that except in the sample roughness, the density function can be very well represented by a decreasing exponential function:

$p=(1 / \lambda) \exp (-x / \lambda)$

where $x$ is the depth in the sample (distance from the bottom of the "roughness" zone at free surface) and $\lambda$ a characteristic length. 


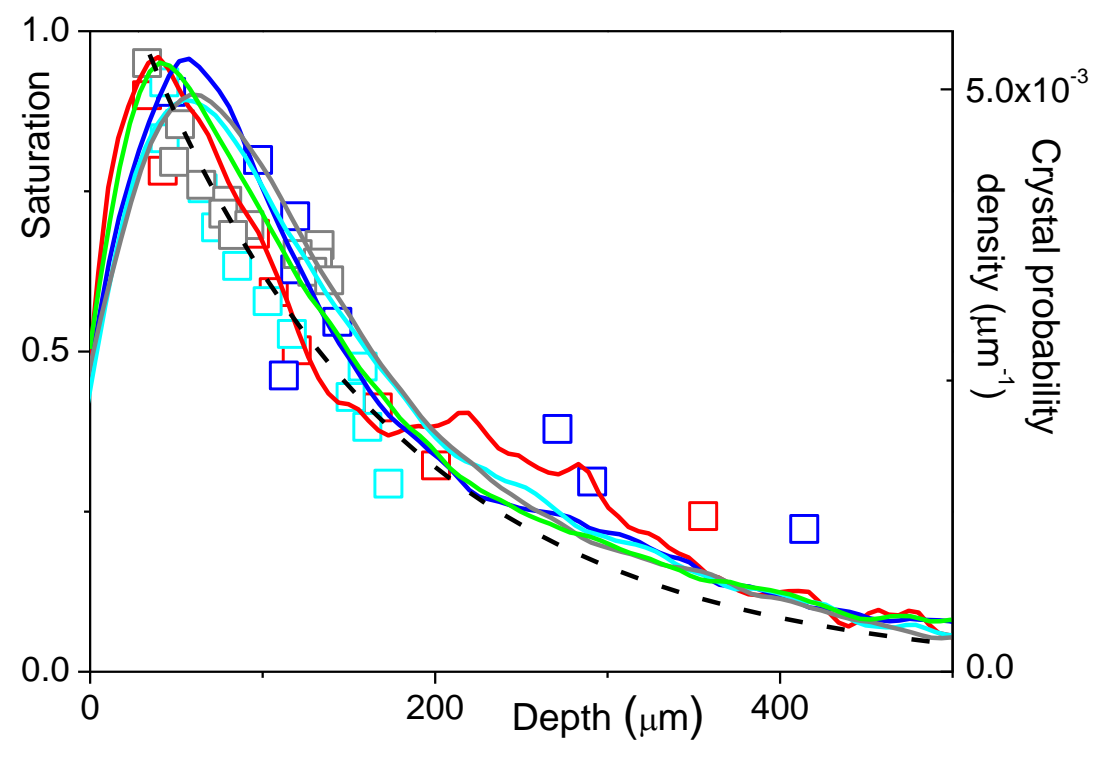

Figure 5: Probability density functions on crystal location estimated from Microtomography as a function of the depth in the sample for a plaster sample after different imbibition-cycles (continuous lines with same colors as in Fig.1). Mean sample saturation (colored squares) as a function of the position of the water front (from MRI profiles, see text). Dashed line: exponential model (equation 1) with $\lambda=150 \mu \mathrm{m}$. Note that for the 4 th cycle the drying could not be observed by MRI due to poor SNR.

This similar distribution of crystals in the sample for the successive imbibition-drying cycles is rather surprising since for each cycle the porous structure evolves and tends to clog further as a result of the deposition of these crystals. From the ion concentration (leading to total crystal mass of $0.055 \mathrm{~g}$ per cycle) and the sample porosity we can compute the effective volume occupied by the crystals. We find that had all the crystals be compacted in the pores they would fill the sample over a thickness of about $40 \mu \mathrm{m}$ after the first drying. This means that for the distribution observed (see Figure 5), extending over a much larger distance, the porosity is far from being filled after the first drying. However it then progressively fills during the next cycles. Indeed after the last cycle the total amount of crystals deposited would saturate the pores over a sample thickness of $200 \mu \mathrm{m}$. Since a large fraction of crystals are in fact distributed in a region with a thickness of this order of magnitude close to the free surface of the sample (see Figure 5) this means that at that time the porosity is very low in this region, which is qualitatively consistent with the results of Figure 4.

Since crystals are expected to deposit mainly around the top liquid-air interface along which most of the evaporation occurs, and since the saturation in the sample decreases as long as crystals deposit, the crystal density decreasing downwards suggests that the liquid interface withdraws (i.e. advances downwards) progressively while leaving crystals behind. It is possible to check this assumption by coming back to the MRI profiles and zooming on the sample top. We effectively see the first air-liquid interface slightly receding in the sample (see insets of Figure $2 a$ and $2 b$ ). This effect will induce a decrease of the drying rate with some analogy with the impact of a dry crust formed after efflorescence [34]. Note that we cannot know the exact extent of the dry region which forms since the profiles correspond to time and space averages, but the progression of the front anyway means that on average the distance between the top liquid-air interfaces and the sample free surface 
increases approximately as deduced from these profiles. For the following computations we arbitrarily consider that the position of the first air-liquid interface is the profile position for an NMR signal of $5 \times 10^{4}$ in our arbitrary units (this is approximately twice the value of the noise on the data). Also note that this recession of the top air-liquid interface does not correspond to a situation of receding front as it occurs in the last stage of drying with hydrophilic material, or immediately after the beginning of drying in a hydrophobic material, when the saturation decreases strictly as a result of the front advance. Here, even if we locally have a slight receding front effect we still have a major effect of homogeneous decrease of the saturation throughout the sample, as shown for example in Figure 2. Finally it is remarkable that the saturation decreases with the front position in a way very similar to the crystal density distribution (see Figure 5), and this evolution may be well described by a similar exponential model:

$$
\phi=\exp (-x / \lambda)
$$

The most striking phenomenon is that all these characteristics are similar for each new cycle: after rescaling them by their integral over the whole thickness (i.e. the expected total amount of crystals at each step) the crystal distribution curves very well superimpose and may be represented by the same exponential model (see Figure 5). That means that successive imbibition-drying cycles simply lead to add a new distribution of crystals of same concentration at each depth in the sample. This is quite surprising since in parallel the apparent drying conditions, namely the drying rate, strongly change (drop of the drying rate in the first regime after each cycle). However if we now turn to the evolution of the interface motion as seen from MRI and represented in terms of saturation as a function of the depth we discover that it is also similar for each cycle although its dynamics obviously strongly differs from one cycle to another and finally covers a two decade range of drying rate. This demonstrates that the position of the receding front only depends on the saturation, and the above law (equation (2)) describing the relationship between the saturation and the front position is general for our material, thus the parameter $\lambda$ is constant in our range of experimental conditions.

This phenomenon is likely explained by a kind of dewetting resulting from crystal formation. It was indeed suggested that different types of interactions of the solution and the crystals with the solid surface can induce different types of processes, such as a recession of the line of contact leading to subflorescence [51]. More precisely it was shown [52] that the drying of $\mathrm{NaCl}$ solution droplets significantly differs from calcium sulfate solution. For the former the crystals formed around the contact line tend to progressively move towards the interior of the droplet as a result of capillary effects, whereas for the latter the crystals forms in the bulk [52-53] but for sufficiently large contact angles of the solution on the solid surface, the crystals remain stuck to the solid surface which finally induces a retraction of the liquid and leads in the end to a "coffee stain effect". Moreover, it was shown, in consistence with our observations, that in drying droplets the crystallization patterns are independent of the drying rate [52]. These observations might be the key explanation of the front recession associated with crystal formation inside plaster.

A consequence of these results is that the front position does not govern by itself the drying rate, since for the same position of this front we observe widely different drying rates for different cycles. It is more likely a change of the diffusion coefficient in the dry region which plays the major role: the vapour coming from the top liquid-air interface and going to the free surface has to find a path through a more tortuous and with smaller pore sizes porous medium as more crystals are deposited in the structure. 


\subsection{Modelling plaster drying}

From these data a simple description of the process emerges. Note that a sophisticated theoretical description allowing to predict salt deposits, water evaporation, stress distribution and damages during this kind of experiment was developed in [41]. Here our approach focuses on salt deposit and water transport and only relies on basic physical assumptions in consistency with above observations. Let us assume that during drying the sample progressively desaturates in the form of a "rectangular" liquid distribution with a decreasing amplitude and decreasing extent: a dry region forms down to an increasing depth $x$, while the saturation $(\phi)$ in the wet region decreases homogeneously. Note that from our data (see insets of Figure 2) we see that this is an approximate description of reality: the front of the liquid distribution is not perfectly straight. For a step of advance of the front $d x$ associated with a change of the mean saturation in the wet region from $\phi$ to $\phi+d \phi$ we have a loss of water volume equal to $d \Omega=-(L-x) S \varepsilon d \phi+d x S \varepsilon \phi$, in which $L$ is the sample height, $S$ its section and $\varepsilon$ its porosity. This leads to the formation of a mass of crystals $d m=m_{0} p d x=\psi d \Omega$, with $m_{0}=\psi S \varepsilon L$ the total mass of crystals that can form in the sample. Since in our case $x<<L$, we can simplify $d \Omega \approx-L S \varepsilon d \phi$ and deduce:

$$
p d x=-d \phi
$$

It is interesting to note that this equation predicts our experimental result concerning the link between $p$ and $\phi$, as it appears from equations (1) and (2) fitted to data, i.e. $p=-d \phi / d x$.

In order to find the law of variation of the saturation or the density function of the crystals we now have to predict the advance of the top liquid-air interface. The above analysis from our data showed that this process mainly depends on the local conditions and not on the drying rate, and it was suggested that it is associated with some dewetting effect. Such a phenomenon depends on the exact shape of the liquid-solid-air interface, which is a priori very complex and in particular depends on the crystal shape which is hardly predictable. We nevertheless assume that this effect can be described in a statistical way in a cross-section of the porous medium so that on average some characteristic step of dewetting would occur after the deposition of some amount of crystals, which may be seen as a number of opportunities of front withdrawal. On the other hand, as the saturation decreases the amount of interfaces to recede decreases. Finally we expect to have an effective withdrawal $d x$ proportional to the volume of crystals deposited between $x$ and $x+d x$, i.e. $d \omega=\left(m_{0} / \rho\right) p d x$ (with $\rho=2300 \mathrm{~kg} \cdot \mathrm{m}^{-3}$ the crystal density), divided by the average cross-section area occupied by the liquid in the pores. We thus obtain $k d \omega / \varepsilon S \phi=d x$, where $k$ is a factor which depends on the physical characteristics of the crystals and the porous structure. We then deduce :

$\phi=(k \psi L / \rho) p$

From (3) and (4) we obtain $\phi d x=-k \psi L d \phi$ which, taking into account the boundary condition $\phi(x=0)=1$, can be integrated and give the expression of the type (2) with

$$
\lambda=k \psi L / \rho
$$

Our model thus predicts the exponential variation of the saturation and the value for the coefficient $k$ may be found from our data and we obtain $k=3.7$. It is interesting to note that $\lambda$ is proportional to the sample height. This means that, although we did not explore that aspect in the present study, the spreading of the crystals in the sample should be larger for longer samples. 
In this context it remains somewhat surprising that we obtain the same law (i.e. equation (2) with the same value for $\lambda$ ) for the successive drying-imbibition cycles whereas the porous structure likely changes as more and more crystals are deposited in a limited volume (which tends to be filled just below the sample free surface). This suggests that $k$ essentially depends on the crystal characteristics rather than on the porous structure. Let us emphasis that $k=1$ would correspond to the formation of a compact crystal layer with the same thickness as that of the current wetting liquid film. By comparison, $k=3.7$ here describes 3.7 times less dense of sparser crystallization.

We can elaborate a simple model predicting the drying rate. As usual we assume that air is saturated with vapor below the first air-liquid interface from the free surface of the sample situated at $x=x_{0}$. When $x_{0}=0$ the interface is in contact with the external air flow and the evaporation may be described in the form of simple diffusion of vapor with a diffusion coefficient $D$ through a boundary layer of thickness $\delta$ [42]. When $x_{0}>0$ we have in addition a diffusion through a porous system with a diffusion coefficient $D(x)=\varepsilon D$ in which $\varepsilon(x)$ is a function of the porous structure which reflects the decrease of porosity and the increase of tortuosity when the crystal concentration increases. In that case the diffusion equation writes $\frac{\partial}{\partial x}\left(D(x) \frac{\partial n}{\partial x}\right)=0$, with $n$ the relative humidity, and the boundary conditions $n(-\delta)=0$ and $n\left(x_{0}\right)=n_{0}$, where $n_{0} \approx 0.96$ is the relative humidity in equilibrium with the saturated $\mathrm{CaSO}_{4}$ solution [54]. The solution for the vapor flux ( $V$ ), expressed in terms of the equivalent volume of liquid water crossing the sample section per unit time, is

$V=n_{0} \frac{D}{\delta} \frac{1}{1+\int_{0}^{x_{0}}(1 / \varepsilon \delta) \mathrm{d} x}$

For the factor $\varepsilon$ which expresses the effect of the new crystals on the tortuosity and porosity, and as a first approximation is inversely proportional to the characteristic length of the path vapor has to follow before reaching the sample free surface, we make the following simple assumption: each new crystal formed (associated to a mass increment $\mathrm{dm}$ ) tends to clog a possible path for vapor which now has to find another path through the rest of the porous structure. This means that the characteristic path length is increased proportionally to the current path length: $d(1 / \varepsilon) \propto(1 / \varepsilon) d m$. We deduce $\varepsilon=\alpha \exp -\left(\beta m_{0} p(x)\right)$, with $m_{0}$ the total (additional) crystal mass in the sample. Note that this approach can be used only when the volume of additional crystals is smaller than the pore volume.

This model appears capable to well predict the shape of the variations of the drying rate in the first regime (i.e. before the strong drying rate decrease) for different initial water to solid ratios by adjusting the only parameter $\alpha$ (see Figure 6), and taking $\lambda$ and $\beta$ as constant. $\alpha$ appears to decrease when the water to solid ratio increases but we have no explanation on the physical origin of this variation.

It is remarkable that with the parameters $\alpha$ and $\beta$ fitted above the model finally reproduces rather well the drying curves (see Fig. 1), not only the decrease during the first stage but also the different plateau levels after each cycle. Thus this model captures the most important physical characteristics of the process. Finally for a low saturation a receding front, of thickness larger than that of the region of accumulation of crystals, starts to develop as a result of a balance capillary and hydrodynamic effects [48] and the second regime of strong decrease of the drying rate is reached (see Fig.1). It also 
appears possible to predict the shape of the drying curve for a sample submitted to different air fluxes (see Figure 7). Note that in that case the initial drying rate taken into account in the model was fitted to the first value in the regime of slow decrease observed just after the initial drop. This initial drop is usually observed in such drying process and is likely due to the disappearance of the thin film of liquid initially covering the sample free surface and the first step of desaturation of the porous medium positioning the first air-liquid interfaces at a depth of the order of a particle radius. However the comparison of model predictions with data for sample of different heights does not show an excellent agreement (see Figure 8). In that case the model parameters are those determined from data of Figure 6 for a sample of height $12 \mathrm{~mm}$ which are directly used to compute model predictions for other heights under similar experimental conditions. Although the shape and decrease of the drying rate curves are qualitatively well predicted there remains a significant quantitative discrepancy (see Figure 8). A possible explanation is that fitting the model to data obtained with small sample heights, and for the first drying process only, might be too sensitive to specific values obtained in that case. Indeed in that case the total crystal amount is much lower than that involved in successive drying-imbibition cycles for large sample heights, and the model fitting is likely more robust.

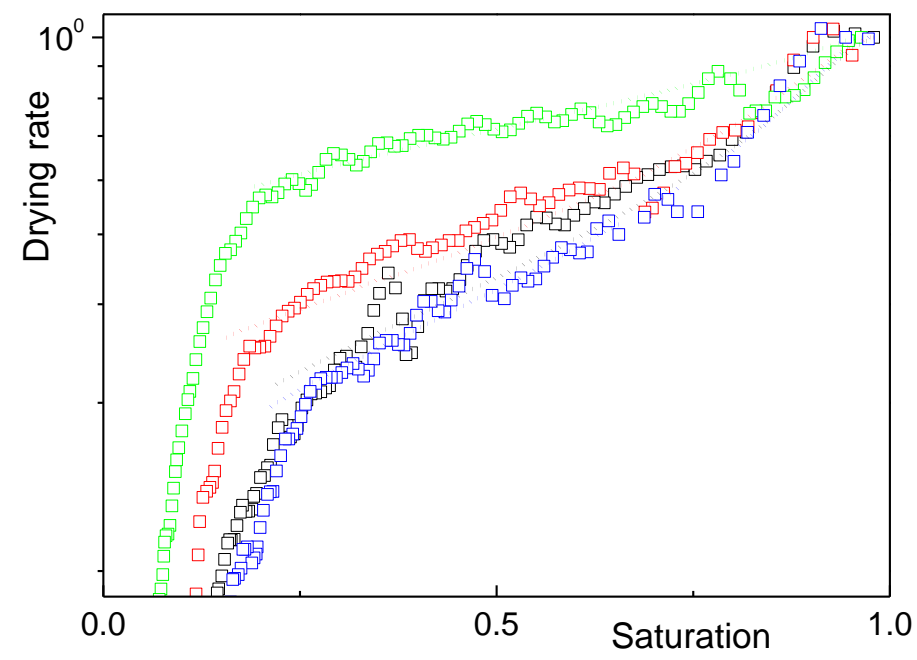

Figure 6: Drying rate (rescaled by the initial one, i.e. $4 \mathrm{~g} / \mathrm{hr}$ ) as a function of saturation and model (equation 6) (continuous line) fitted to data for plaster (first drying after preparation) at different initial water to powder ratios (sample height $12 \mathrm{~mm}$ ) (data from [13]): 0.8 (green), $\alpha=48 \times 10^{4}$, 0.7 (red), $\alpha=11.5 \times 10^{4}, 0.6$ (black), $\alpha=8.6 \times 10^{4}, 0.5$ (blue), $\alpha=6.7 \times 10^{4}$, with $\beta=4000 \mathrm{~g}^{-1}$ for all materials. 


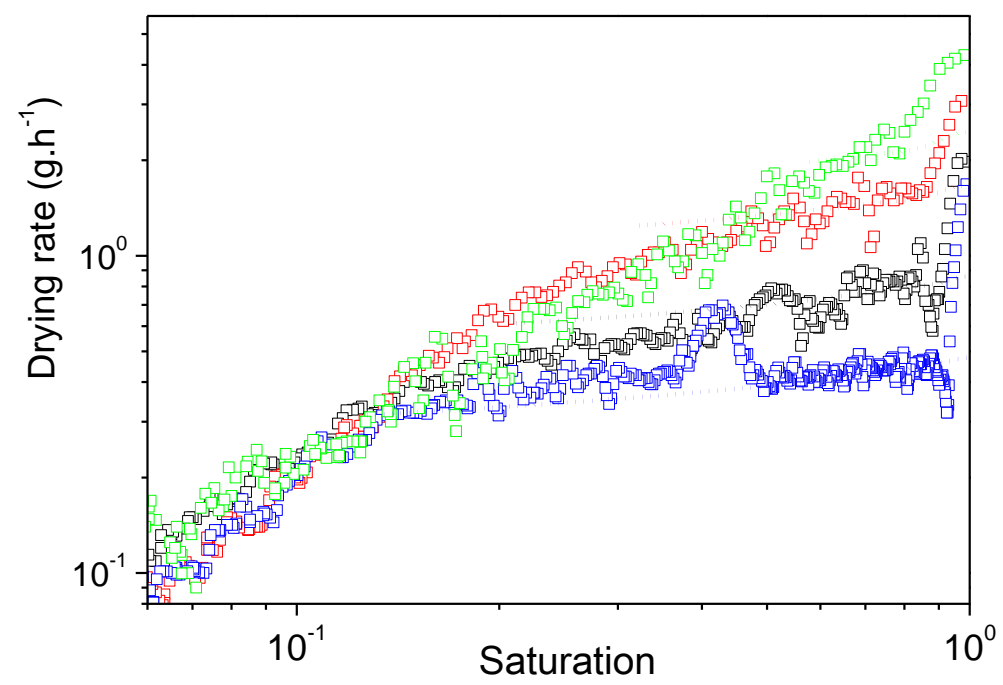

Figure 7: Drying rate as a function of saturation and model (equation 6) (continuous line) fitted to data for plaster (first drying after preparation) (with $\alpha=8.6 \times 10^{4}$ and $\beta=4000 \mathrm{~g}^{-1}$ ) for an initial water to powder ratio of 0.6 (data from [13]) at different velocities of the air flux (sample height $12 \mathrm{~mm}$ ): $7.3 \mathrm{~m} . \mathrm{s}^{-1}$ (green), $2.6 \mathrm{~m} . \mathrm{s}^{-1}$ (red), $1.2 \mathrm{~m} . \mathrm{s}^{-1}$ (black), 0 (blue).

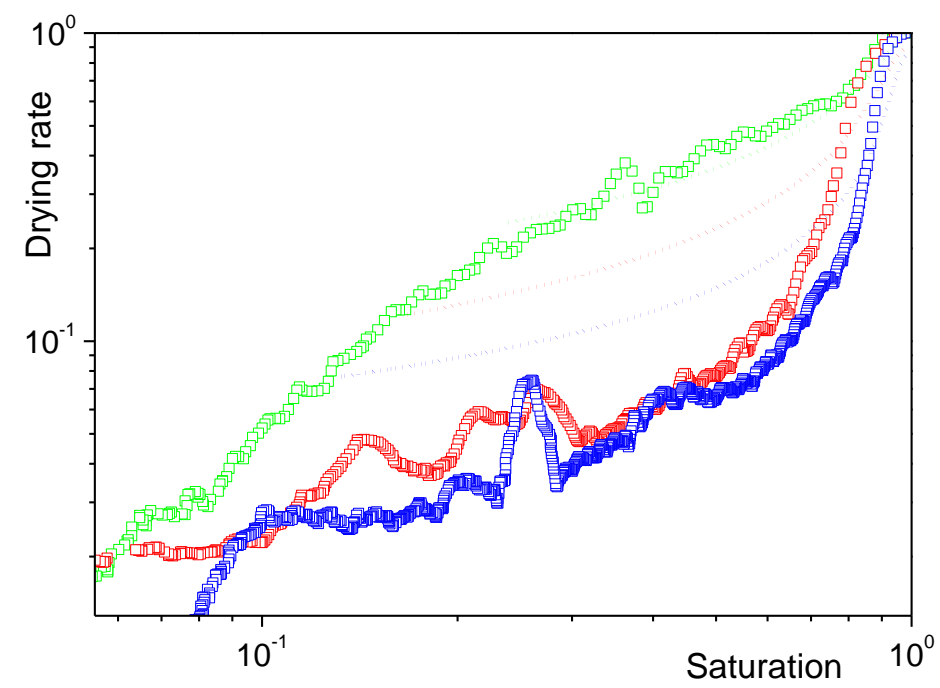

Figure 8: Drying rate (rescaled by the initial one, i.e. $3.4 \mathrm{~g} / \mathrm{hr}$ ) as a function of saturation, and model (equation 6, with $\alpha=8.6 \times 10^{4}$ and $\beta=4000 \mathrm{~g}^{-1}$ ) (continuous line) fitted to data for plaster (first drying after preparation) with initial water to powder ratio 0.6 and different sample heights: $12 \mathrm{~mm}$ (green), $22 \mathrm{~mm}$ (red); $40 \mathrm{~mm}$ (blue).

\section{Conclusion}

This work shows that it is possible to quantify, on average, the effects which occur at a local scale inside the sample in terms of water transport and crystal formation, which then allow to understand 
the dynamics of drying coupled with crystallization in porous media. The simple physical mechanics observed provides a full frame for describing the drying properties of plaster, and are likely applicable to other situations. However the parameters of the model, i.e. $\lambda, \alpha$ and $\beta$ depend on the characteristics of the ions and the porous structure, and their exact physico-chemical origin would need to be studied with the help of experiments at a local scale. Our approach nevertheless provides some basic understanding of subflorescence dynamics in building materials, and suggests that it should be possible to avoid subflorescence by maintaining a wetting up to the sample free surface with appropriate additives in the liquid.

\section{References}

1. Neville HA. Adsorption and Reaction I. J Phys Chem 1926;30:1037-1042.

2. Ridge MJ, Beretka J. Calcium Sulphate Hemihydrate and Its Hydration. Rev Pure and Appl Chem 1969;19:17-44.

3. Hansen WC. Setting and hardening of gypsum plaster. Mat Res and Standards. 1963;3:359-363.

4. Vignes JL, Essaddam H. Une vie de plâtre. Bulletin de l'union des physiciens. 1997;790:145-164.

5. Singh NB, Middendorf B. Calcium sulphate hemihydrate hydration leading to gypsum crystallization. Progress in Crystal Growth and Characterization of Materials. 2007 ;53:57-77.

6. Lemarchand A, Boudoire F, Boucard E, Chotard T, Smith A. Plaster Hydration at Different Plasterto-Water Ratios: Acoustic Emission and 3-Dimensional Submicrometric Simulations. The Journal of Physical Chemistry. 2012;116:4671-4678.

7. Jaffel H, Korb JP. Probing Microstructure Evolution during the Hardening of Gypsum by Proton NMR Relaxometry. J. Phys. Chem. B. 2006;110:7385-7391.

8. Frederiksen R, Marchand E (Eds). Plaster casts: making, collecting and displaying from classical antiquity to the present. De Gruyter Berlin. 2010.

9. Petkovic J, Huinink HP, Pel L, Kopinga K, van Hees RPJ, Salt transport in plaster/substrate layers. Mat and Struct. 2007;40:475-490.

10. Vosten B, Hersfeld B. The drying of crust-forming materials as exemplified by the drying of gypsum wallboard (in German). Zement-Kalk-Gips. 1976; 5:213-222.

11. Song KM, Mitchell J, Gladden LF. Magnetic resonance studies of hydration kinetics and microstructural evolution in plaster pastes. J Mater. Sci. 2009;44:5004-5012.

12. Song KM, Mitchell J, Jaffel H, Gladden LF. Simultaneous monitoring of hydration kinetics, microstructural evolution, and surface interactions in hydrating gypsum plaster in the presence of additives. J Mater. Sci. 2010; 45: 5282-5290.

13. Seck MD, Van Landeghem M, Faure $P$, Rodts $S$, Combes $R$, Cavalié $P$, Coussot $P$. The mechanisms of plaster drying. J. Mat. Sci. 2015;50:2491-2501.

14. Liu Z, Deng D, De Schutter G. The damage of calcium sulfoaluminate cement paste partially immersed in MgSO4 solution. Constr and build mater. 2014;66:692-711.

15. Sawdy A, Heritage A, Pel L. A review of salt transport in porous media, assessment methods and salt reduction treatments. In Proceedings International Conference on Salt Weathering on Buildings and Stone Sculptures, Technical University of Denmark. 2008;Lyngby 1-28.

16. Peysson Y, Bazin B, Magnier C, Kohler E, Youssef S. Permeability alteration due to salt precipitation driven by drying in the context of CO2 injection. Energy Proc. 2011;4:4387-4394.

17. Rodriguez-Navarro C, Doehne E. Salt Weathering: Influence of Evaporation Rate, Supersaturation and Crystallization Pattern. Earth Surf. Process. Landforms. 1999;24:191-209. 
18. Espinoza-Marzal RM, Scherer GW. Impact of in-pore salt crystallization on transport properties. Environ. Earth Sci. 2012;69:2669.

19. Kramar S, Urosevic M, Pristacz H, Mirtic B. Assessment of limestone deterioration due to salt formation by micro-Raman spectroscopy: application to architectural heritage. J Raman Spectrosc. 2010;41:1441-1448.

20. Foraboschi $P$, Vanin A. Experimental investigation on bricks from historical Venetian buildings subjected to moisture and salt crystallization. Eng. Failure Analysis. 2014;45:185-203.

21. Scherer GW. Stress from crystallization of salt. Cement Concrete Res. 2004;34:1613-1624.

22. Flatt RJ. Salt damage in porous materials: how high supersaturations are generated. J. Crystal Growth. 2002;242(3):435-454.

23. Schiro M, Ruiz-Agudo E, Rodriguez-Navarro C. Damage Mechanisms of Porous Materials due to In-Pore Salt Crystallization. Phys. Rev. Lett. 2012;109:265503.

24. Flatt RJ, Caruso F, Aguilar Sanchez AM, Scherer GW. Chemo-mechanics of salt damage in stone. Nature Communications 2014;5:4823.

25. Tsui N, Flatt RJ, Scherer GW. Crystallization damage by sodium sulfate. J. Cultural Heritage. 2003;4:109-115.

26. Espinosa-Marzal RM, Scherer GW. Advances in Understanding Damage by Salt Crystallization. Accounts of Chemical Research. 2010;43:897-905.

27. Schultz $P$, Schlünder EU. Influence of additives on crust formation during drying. Chem. Eng. Process. 1990;28:133-142.

28. Shahidzadeh-Bonn N, Desarnaud J, Bertrand F, Chateau X, Bonn D, Damage in porous media du to salt crystallization, Phys. Rev. E. 2010;81:066110

29. Desarnaud J, Bertrand F, Shahidzadeh-Bonn N. Impact of the kinetics of salt crystallization on stone damage during rewetting/drying and humidity cycling. J. Appl. Mech. 2013;80:020911.

30. Pel L, Huinink H, Kopinga K, van Hees RPJ, Adan OCG. Efflorescence pathway diagram: understanding salt weathering. Constr Build Mater. 2004;18:309-313.

31. Petkovic J, Huinink HP, Pel L, Kopinga K, van Hees RPJ. Moisture and salt transport in three-layer plaster/substrate systems. Construction and Building Materials. 2010;24:118-127.

32. Veran-Tissoires S, Marcoux M, Prat M. Discrete Salt Crystallization at the Surface of a Porous Medium. Phys. Rev. Lett. 2012;108:054502.

33. Sghaier N, Prat M. Effect of efflorescence formation on drying kinetics of porous media. Transp. Porous Med. 2009;80:441-454.

34. Desarnaud J, Derluyn H, Molari L, de Miranda S, Cnudde V, Shahidzadeh N. Drying of salt contaminated porous media: Effect of primary and secondary nucleation. J. Appl. Phys., 2015;118:114901.

35. Norouzi Rad M, Shokri N, Kesmiri A, Withers PJ. Effects of grain and pore size on salt precipitation during evaporation from porous media: A pore-scale investigation. Transp. Porous Med. 2015;DOI: 10.1007/s11242-015-0515-8.

36. Gupta S, Huinink HP, Pel L, Kopinga K. How ferrocyanide influences $\mathrm{NaCl}$ crystallization under different humidity conditions. Crystal Growth Design. 2014;14:1591-1599.

37. S. Gupta, H.P. Huinink, M. Prat, L. Pel, K. Kopinga. Paradoxical drying of a fired-clay brick due to salt crystallization. Chem. Eng. Sci. 2014;109:204.

38. Shokri N. Pore-scale dynamics of salt transport and distribution in drying porous media. Phys. Fluids. 2014;26:012106.

39. Sghaier N, Geoffroy S, Prat M, Eloubaki H, Ben Nasrallah S. Evaporation-driven growth of large crystallized salt structures in a porous medium. Phys. Rev. 2014;90:042402 
40. Derluyn H., Griffa M, Mannes D, Jerjen I, Dewanckele J, Vontobel P, Sheppard A, Derome D, Cnudde V, Lehmann E, Carmeliet J. Characterizing saline uptake and salt distributions in porous limestone with neutron radiography and X-ray micro-tomography. J. Building Physics 2013;36:353374.

41. Derluyn $H$, Moonen $P$, Carmeliet J. Deformation and damage due to drying-induced salt crystallization in porous limestone. Journal of the Mechanics and Physics of Solids. 2014;63:242255.

42. Keita $E$, Faure $P$, Rodts $S$, Coussot P. MRI evidence for a receding-front effect in drying porous media. Phys. Rev. E. 2014;87:062303.

43. Jaffel H. Multi-scale characterization of evolving porous media (in French). Ph.D. thesis. Ecole Polytechnique, Palaiseau, 2009.

44. Carr H, Purcell E. Effects of Diffusion on Free Precession in Nuclear Magnetic Resonance Experiments. Phys. Rev. 1954;94:630.

45. Meiboom S, Gill D. Modified SpinEcho Method for Measuring Nuclear Relaxation Times. Rev. Sci. Instrum.1958;29:688.

46. McDonald PJ, Korb JP, Mitchell J, Monteilhet L. Surface relaxation and chemical exchange in hydrating cement pastes: a two-dimensional NMR relaxation study. Phys. Rev. E. 2005;72:011409.

47. Van Brakel J. Mass transfer in convective drying. Adv. Drying. 1980;1:217-267.

48. Coussot P. Scaling convective drying. Eur. Phys. J. E. 2000;15:557-566.

49. Mantellato S, Palacios M, Flatt RJ. Reliable specific surface area measurements on anhydrous cements. Cement and Concrete Research 2015;67: 286-291.

50. Faure P, Coussot P. Drying of a model soil. Phys. Rev. E. 2010;82:036303.

51. Shahidzadeh-Bonn N, Rafaï S, Bonn D, Wegdam G. Salt crystallization during evaporation: impact of interfacial properties. Langmuir. 2008;24:8599-8605.

52. Shahidzadeh N, Schut MFL, Desarnaud J, Prat M, Bonn D. Salt stains from evaporating droplets. Scientific Reports. 2015;5:10335.

53. Wang, Y.W., H.K. Christenson, F.C. Meldrum, Confinement leads to control over Calcium Sulfate Polymorph. Adv. Funct. Mater. 2013;23:5615-5623

54. Berdugo, I., Romero E., Saaltink M., Albis M. On the behaviour of the Ca-SO4-H2O system. Rev. Acad. Colomb. Cienc. 2008;32(125): 545-557. 